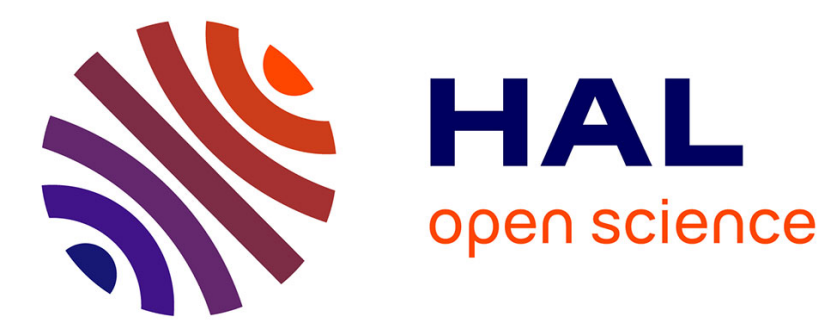

\title{
Induced Anisotropy in Foams Forming Processes: Modelling and Simulation
}

Francisco Chinesta, Elías Cueto, Peregrina Quintela, Jesus Paredes

\section{To cite this version:}

Francisco Chinesta, Elías Cueto, Peregrina Quintela, Jesus Paredes. Induced Anisotropy in Foams Forming Processes: Modelling and Simulation. Journal of Materials Processing Technology, 2004, 155-156, pp.1482-1488. 10.1016/j.jmatprotec.2004.04.378 . hal-00020773

\section{HAL Id: hal-00020773 https://hal.science/hal-00020773}

Submitted on 26 Mar 2018

HAL is a multi-disciplinary open access archive for the deposit and dissemination of scientific research documents, whether they are published or not. The documents may come from teaching and research institutions in France or abroad, or from public or private research centers.
L'archive ouverte pluridisciplinaire HAL, est destinée au dépôt et à la diffusion de documents scientifiques de niveau recherche, publiés ou non, émanant des établissements d'enseignement et de recherche français ou étrangers, des laboratoires publics ou privés. 


\title{
Induced anisotropy in foams forming processes: modelling and simulation
}

\author{
F. Chinesta ${ }^{\mathrm{a}, *}$, E. Cueto $^{\mathrm{b}}$, P. Quintela ${ }^{\mathrm{c}}$, J. Paredes $^{\mathrm{c}}$ \\ ${ }^{a}$ LMSP, UMR 8106 CNRS-ENSAM-ESEM, 151 Boulevard de l'Hôpital, F-75013 Paris, France \\ b Aragón Institute for Engineering Research (I3A), University of Zaragoza, María de Luna, 3, E-50018 Zaragoza, Spain \\ ${ }^{c}$ Departamento de Matemática Aplicada, Universidad de Santiago de Compostela, 15706 Santiago de Compostela, Spain
}

\begin{abstract}
The numerical modelling of forming processes involving the flow of foams requires taking into account the different problem scales. Thus, in industrial applications a macroscopic approach is suitable, whereas the macroscopic flow parameters depend on the cellular structure: porosity, size, shape and orientation of the cells, cellular walls properties, etc. Moreover, the shape and orientation of the cells are induced by the flow during the foam expansion (induced anisotropy). This work focuses on this topic, covering both the mechanical and the numerical modelling. In particular we will analyse two kind of numerical approaches: the first one involving a fixed mesh of the whole domain (which induces some numerical difficulties related to the advection of the free surface and other fields). The second approach lies in the use of a meshless technique which allows accurately both fields advection and flow front tracking, without a mesh support. The fundamentals of this work are based on some of our former works cited in the references section.
\end{abstract}

Keywords: Numerical modelling; Foam forming processes; Induced anisotropy; Multiscale approach

\section{Introduction}

Reactive systems are widely used in foam blowing processes. Numerical modelling of reactive processes is very difficult because the flow kinematics is coupled with the chemical reacting kinetics, which depends mainly on the reaction time (time elapsed since the reactants mixing). The kinematics-chemical kinetics coupling is a key point in foam blowing, being less important, for example, in usual RTM (resin transfer moulding) processes, where the chemical formulation can be adjusted in order to finish the mould filling before a significant rise in the resin viscosity.

The polymerisation reaction increases the material viscosity and the expansion induces the material growing, generating a porous structure. When the polymerisation takes place much faster than the foam expansion, the material becomes too rigid before the conclusion of the expansion. In this case the cellular walls can be broken with direct consequences in the mechanical properties of the conformed parts. On the other hand, when the expansion finishes before the complete polymerisation, the low material consistency is not enough

\footnotetext{
* Corresponding author.

E-mail addresses: francisco.chinesta@paris.ensam.fr (F. Chinesta), ecueto@unizar.es (E. Cueto), pere@zmat.usc.es (J. Paredes).
}

to preserve the final geometry, and the structure collapses when the expansion is finished.

The numerical modelling of forming processes involving the flow of foams requires taking into account the different problem scales. Thus, in industrial applications a macroscopic approach is suitable, whereas the macroscopic flow parameters depend on the cellular structure: porosity, size, shape and orientation of the cells, cellular walls properties, etc. Moreover, the shape and orientation of the cells are induced by the flow during the foam expansion (induced anisotropy). We have analysed the influence of process conditions on the cellular shape [1]: elongated cells were obtained by inducing traction or shear in the material during its expansion.

This microscopic information can be introduced in a macroscopic model by means of a homogenisation technique. Thus, at each point in the material domain we can associate a representative volume containing some cells, whose size, shape and orientation depend on the considered point. Now, from the matrix and gas viscosities, and the cellular structure, we can compute an equivalent viscosity tensor (see Fig. 1). However, some difficulties appear in the homogenisation procedure: (i) sometimes surface tension cannot be neglected, which requires a very accurate description of the inclusion geometry as well as to consider 


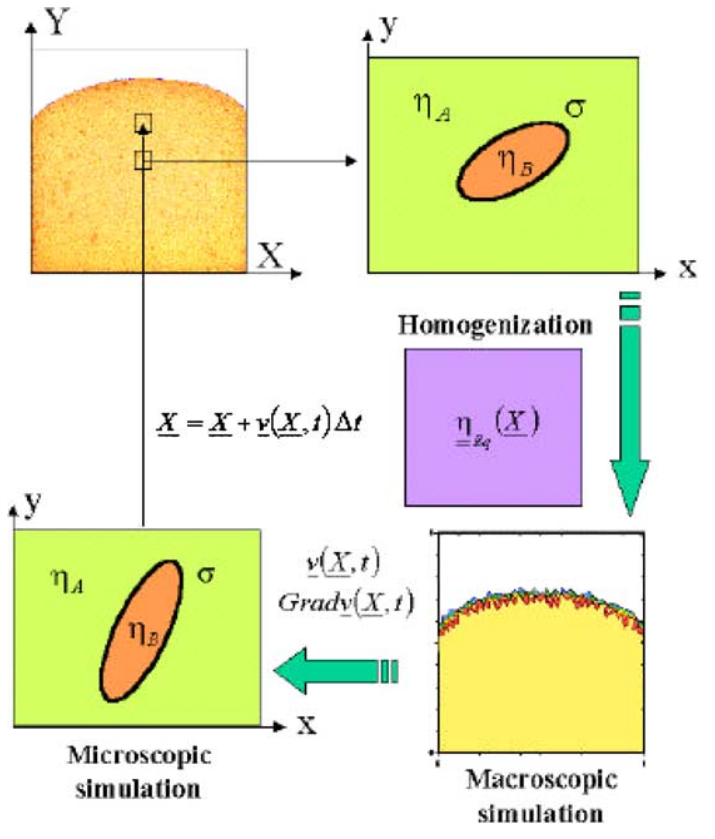

Fig. 1. Micro-macro simulation.

its effect on the homogenised model; (ii) the behaviour of the matrix fluid becomes quickly non-Newtonian, and its non linear character makes difficult the application of usual homogenisation techniques; (iii) the reaction kinetics remain uncertain for usual industrial processes; (iv) the numerical tracking of a cell (microscopic level) which grows and deforms into the material flow is today an open problem, which in spite of some promising and recent works [2-7] important difficulties persist; (v) the physical mechanism of gas diffusion, the cells interaction and fusion, and the modelling of the final stage of the process, when the material is concentrated on the cell walls, are, among many others, important and unsolved difficulties found in the global modelling of foam growing.

In this way a macroscopic modelling where the reaction kinetics and the viscosity evolution are obtained experimentally is suitable from the point of view of its industrial application to provide a visualisation of the mould filling process (foam volume evolution and flow front position during the expansion) and it will be useful to optimise the mould and process design. From that simulation we can determine the number, size and optimal position of the evacuation orifices to avoid internal overpressures as well as to reduce the cleaning necessities and the material losses. Moreover, an accurate flow front tracking allows us to predict the interactions between different flow fronts when the curing reaction is too advanced (welding lines prediction) as well as to avoid filling defects related to the air bubbles retained between the flow front and the mould walls.

In this way the process simulation must be carried out in two different scales. In the macroscopic scale the flow kinematics is computed and the expansion flow front and the foam volume are updated according to that kinematics. On the other hand, the microstructure evolution must be computed in the microscopic scale. This computation, local in space, makes use of the macroscopic kinematics. In this paper, we assume an expansion function experimentally identified for each reacting system as described in [8], which avoids the necessity of considering the diffusion mechanisms in the cellular growth, which are not well known at present. Thus, from now on, we consider the expansion function $\beta(t)$ known

$\beta(t)=-\frac{\partial \rho}{\partial t} \frac{1}{\rho}$

From the numerical simulation point of view two possibilities exist. The first one is based on the use of a fixed mesh to solve the mechanical problems at both scales, and the other one that lies on the use of a moving mesh. The main drawback of the first approach is the accurate description of moving boundaries and interfaces. Some appealing strategies for treating this kind of problems are the extended finite element method [6,7], the volume of fluid method [9], the level set technique [2,5], among many others. However, as described later, these capturing techniques require solving some hyperbolic transport problems, which introduce some important numerical difficulties. On the other hand, tracking techniques adjust the mesh to the domain evolution, and this fact introduces, in the context of the finite element method, some problems related to large mesh distortions. Recently, some meshless methods have been introduced (see [10] for a brief overview) which reduce drastically the geometric mesh requirements. Among all these techniques, an appealing strategy is the natural element method, which allows large domain evolutions without any remeshing requirement, as well as the direct imposition of essential boundary conditions in any geometry (convex or not) [11-13]. The application of both kinds of strategies in multiscale foam expansion simulations will be treated in Sections 3 and 4, using the mechanical models described in the next section. The results presented in this work concern some test problems defined in the micro and in the macro scales, but a real coupling between both problem scales, according to the procedure illustrated in Fig. 1, is a work in progress, out of the aim of this paper.

\section{Mechanical modelling}

\subsection{Macroscopic model}

The equations modelling the foam kinematics during the expansion are given by the usual balance equations (where all thermal effects are neglected):

- The equilibrium equation neglecting the inertia and mass terms results

$\operatorname{Div} \underline{\underline{\sigma}}=\underline{0}$

where $\underline{\underline{\sigma}}$ is the stress tensor. 
- The mass conservation

$\frac{\partial \rho}{\partial t}+\operatorname{Div}(\rho \underline{v})=0$

where $\underline{v}$ is the velocity field and $\rho$ the foam density. The previous equation can be rewritten as

$\frac{\partial \rho}{\partial t}+\rho \operatorname{Div} \underline{v}+\underline{v} \operatorname{Grad} \rho=0$

When the foam expansion is homogeneous $\rho=\rho(t)$ and $\operatorname{Grad} \rho=\underline{0}$. In this case, Eq. (4) becomes

$\operatorname{Div} \underline{v}=-\frac{\partial \rho}{\partial t} \frac{1}{\rho}=\beta(t)$

- A simplified constitutive equation is given by

$\underline{\underline{\sigma}}=-p \underline{\underline{I}}+\left(\lambda-\frac{2}{3} \eta\right) \operatorname{Tr}(\underline{\underline{D}}) \underline{\underline{I}}+2 \eta \underline{\underline{D}}$

where $\lambda$ denotes the volumetric viscosity, $p$ the pressure field, $\underline{I}$ the unit tensor, $\eta$ the shear viscosity and $\underline{D}$ the strain rate tensor (symmetric part of the gradient of velocity tensor). If the volumetric viscosity contribution is neglected, it results

$\underline{\underline{\sigma}}=-p \underline{\underline{I}}+2 \eta \underline{\underline{D}}$

where $\underline{D}$ denotes in this case the deviatoric strain rate tensor. In the examples shown in this paper a homogenised local viscosity has been considered. See [14-16], and the references therein, for a depth discussion on foam rheology.

\subsection{Microscopic model}

In the microscopic scale the heterogeneity of the flow must be introduced. Thus, we consider a representative domain containing some cells into a polymer matrix, whose averaged kinematics is derived locally from the macroscopic kinematics. At this stage, the cell growth can be computed using the expansion function, and the evolution of their shapes and orientations requires the resolution of a flow problem in the representative flow domain. In this way, the microscopic flow problem results similar to that one involving a bubble immersed in a Newtonian fluid. The numerical modelling must consider the existence of two fluids, whose domains evolve in time, with an eventual surface tension on its interface. Thus, some results involving the deformation of a bubble into a Newtonian fluid will be introduced later.

\section{Fixed mesh simulation}

\subsection{Macroscopic level}

The variational formulation of Eqs. (2) and (5) and Eq. (7) results: Find $\underline{v} \in H^{1}(\Omega(t))$ verifying the essential boundary conditions $\underline{v}\left(\underline{x} \in \partial_{1} \Omega(t)\right)=\underline{v}_{\mathrm{g}}$ and $p \in L^{2}(\Omega(t))$ such that

$\int_{\Omega(t)}\left(-p \operatorname{Tr}\left(\underline{\underline{D}}^{*}\right)+2 \eta \underline{\underline{D}}: \underline{\underline{D}}^{*}\right) \mathrm{d} \Omega=0$ and

$\int_{\Omega(t)}-p^{*}(\operatorname{Div} \underline{v}-\beta) \mathrm{d} \Omega=0$

$\forall \underline{v}^{*} \in H_{0}^{1}(\Omega(t))$ and $\forall p^{*} \in L^{2}(\Omega(t))$

where a null traction is assumed in $\partial_{2} \Omega(t)=\partial \Omega(t)-\partial_{1} \Omega(t)$, a homogeneous expansion is considered $(\operatorname{Grad} \rho=0$ in Eq. (4)), and $H^{1}(\Omega(t)), H_{0}^{1}(\Omega(t)), L^{2}(\Omega(t))$ are the usual Sobolev and Lebesgue functional spaces.

In order to update the foam volume, some possibilities exist. The simplest lies in the introduction of a foam presence function $I$ that takes a unit value in the foam domain and vanishes in the empty region, i.e.

$I(\underline{x}, t)= \begin{cases}1, & \underline{x} \in \Omega(t), \\ 0, & \underline{x} \notin \Omega(t)\end{cases}$

and whose evolution is governed by the following linear advection equation:

$\frac{\partial I}{\partial t}+\underline{v} \operatorname{Grad} I=0$

The discretisation of Eqs. (8) and (9) is carried out using a mixed finite element formulation. The mini-element $C^{0}-P_{1+\text { bubble }}$ is used for the velocity interpolation whereas a $C^{0}-P_{1}$ approximation is considered in the pressure interpolation. This choice verifies the LBB condition [17]. On the other hand, due to the hyperbolic character of Eq. (11) a discontinuous Galerkin finite element method is considered. The conservation form of Eq. (11) is:

$\int_{\Omega^{\mathrm{e}}}\left(\frac{\partial I}{\partial t}+\operatorname{Div}(I \underline{v})-I \operatorname{Div} \underline{v}\right) \mathrm{d} \Omega=0$

where $\Omega^{\mathrm{e}}$ represents an element of the finite element mesh associated with the foam domain $\Omega(t)$. Eq. (12) can be written as

$$
\begin{gathered}
\int_{\Omega^{\mathrm{e}}} \frac{\partial I}{\partial t} \mathrm{~d} \Omega+\int_{\partial \Omega^{\mathrm{e}+}} I \underline{v} \cdot \underline{n} \mathrm{~d} S+\int_{\partial \Omega^{\mathrm{e}-}} I \underline{v} \cdot \underline{n} \mathrm{~d} S \\
-\int_{\Omega^{\mathrm{e}}} I \operatorname{Div} \underline{v} \mathrm{~d} \Omega=0
\end{gathered}
$$

where $\partial \Omega^{\mathrm{e}^{+}}$and $\partial \Omega^{\mathrm{e}^{-}}$denote the outflow and inflow boundaries of the element $\Omega^{\mathrm{e}}$, respectively. The main difficulty of using Eq. (13) is that the function $I$ is not defined on the element boundaries. The discontinuous Galerkin finite element method assumes that on the outflow boundary, $I$ takes the existing value in the element $\Omega^{\mathrm{e}}$, i.e. $I\left(\underline{x} \in \partial \Omega^{\mathrm{e}^{+}}\right)=I^{\mathrm{e}}$ (the function $I$ is assumed constant into each element); and that on the inflow boundary $I$ takes the existing value in the upstream element. Thus, Eq. (13) can be written in the case of a homogeneous expansion in the form

$$
\begin{aligned}
& \frac{\partial I^{\mathrm{e}}}{\partial t}\left|\Omega^{\mathrm{e}}\right|=-I^{\mathrm{e}} \int_{\partial \Omega^{\mathrm{e}+}} \underline{v} \cdot \underline{n} \mathrm{~d} S-\sum_{i} I^{\mathrm{e}_{i}^{-}} \int_{\partial \Omega^{\mathrm{e}_{i}^{-}}} \underline{v} \cdot \underline{n} \mathrm{~d} S \\
& \quad+I^{\mathrm{e}} \beta\left|\Omega^{\mathrm{e}}\right|
\end{aligned}
$$


where the sum is extended to the neighbouring upstream elements of $\Omega^{\mathrm{e}}, \Omega^{\mathrm{e}^{-}}$, whose value of $I$ is represented by $I^{\mathrm{e}_{i}^{-}}$; Div $\underline{v}$ is done by Eq. (5), and $\left|\Omega^{\mathrm{e}}\right|$ denotes de volume of $\Omega^{\mathrm{e}}$. To simplify the notation, we will consider, from now on, that the flow coming to the element $\Omega^{\mathrm{e}}$ comes from only one element. If we consider a first order explicit approximation of the time derivative, we can write Eq. (14) as

$I^{\mathrm{e}}(t+\Delta t)=I^{\mathrm{e}}-I^{\mathrm{e}} \frac{q^{+} \Delta t}{\left|\Omega^{\mathrm{e}}\right|}+I^{\mathrm{e}^{-}} \frac{q^{-} \Delta t}{\left|\Omega^{\mathrm{e}}\right|}+I^{\mathrm{e}} \beta \Delta t$

where $q^{+}$and $q^{-}$are the absolute value of the outflow and inflow rates, respectively, and all the terms in the second member are evaluated at time $t$ (explicit strategy). Now, we define the inflow and outflow fluid volumes as $\Omega^{-}=q^{-} \Delta t$ and $\Omega^{+}=q^{+} \Delta t$, respectively. Thus, we can write finally

$I^{\mathrm{e}}(t+\Delta t)=I^{\mathrm{e}}-I^{\mathrm{e}} \frac{\Omega^{+}}{\left|\Omega^{\mathrm{e}}\right|}+I^{\mathrm{e}^{-}} \frac{\Omega^{-}}{\left|\Omega^{\mathrm{e}}\right|}+I^{\mathrm{e}} \beta \Delta t$

Eq. (16) allows the foam domain updating, considering that the fluid domain is defined by the set of elements having a value of $I$ greater than a threshold value. It can be noticed that the exact flow front position cannot be determined accurately. An accurate treatment of the transport of other fields, which are not defined in the empty region, was proposed in [18]. It is based on the application of the previous explicit first-order technique to the product $I F$ where $F$ is an arbitrary field related to the fluid and advected by the flow. In Fig. 2 the foam presence function evolution during a homogeneous expansion in a closed square cavity is shown.

\subsection{Microscopic level}

The main difficulty in applying the previous modelling for treating the microscopic problem is related to the low accuracy in the interface location. In this form, the accurate imposition of a surface tension, for example, is not possible. To alleviate this problem, one possibility lies on the application of the level set strategy in order to locate the moving interfaces. This technique is based on the substitution of the fluid presence function by a level set function $\phi$ whose zero value defines the interface. If we denote by $\Omega_{\mathrm{p}}(t)$ and $\Omega_{\mathrm{g}}(t)$ the polymer and the gas domains at time $t$, respectively, and by $\Gamma(t)$ the moving interface $\left(\Gamma(t)=\overline{\Omega_{\mathrm{p}}(t)} \cap \overline{\Omega_{\mathrm{g}}(t)}\right)$, the level set function is given by

$\phi(\underline{x}, t) \begin{cases}>0, & \underline{x} \in \Omega_{\mathrm{p}}(t), \\ <0, & \underline{x} \in \Omega_{\mathrm{g}}(t), \\ =0, & \underline{x} \in \Gamma(t)\end{cases}$

Obviously, the level function is convected by the flow, and then, its evolution is given by the following linear advection equation:

$\frac{\partial \phi}{\partial t}+\underline{v}^{*} \operatorname{Grad} \phi=0$

where $\underline{v}^{*}$ is an arbitrary velocity field, verifying

$\underline{v}^{*}(\underline{x} \in \Gamma(t))=\underline{v}(\underline{x} \in \Gamma(t))$

It has been proved by several authors that the quality of the level function decreases during its time evolution. In order to control the excessive numerical diffusion, which destroys the sharpness of the front, some reinitialisation processes must be introduced to maintain the level set function as a distance function for all times. Thus, from a solution $\phi(\underline{x}, t)$, a distance function $\tilde{\phi}(\underline{x}, t)$ can be computed from the steady solution of the following non-linear advection equation:

$\frac{\partial \tilde{\phi}}{\partial \tau}+\operatorname{sgn}(\phi) \frac{\operatorname{Grad} \tilde{\phi}}{\|\operatorname{Grad} \tilde{\phi}\|} \operatorname{Grad} \tilde{\phi}=\operatorname{sgn}(\phi)$

where the initial condition is given by

$\tilde{\phi}(\underline{x}, \tau=0)=\phi(\underline{x}, t)$

and where $\tau \in[0, \infty[$ is an artificial time and sgn is the sign function. When the corrected function $\tilde{\phi}(\underline{x}, t)$ is known, we assign this function to the level set function $(\tilde{\phi}(\underline{x}, \tau=\infty) \rightarrow$ $\phi(\underline{x}, t))$ in order to update its value from Eq. (18) at the next time step. The density and viscosity can be expressed from the densities and viscosities of polymer and gas:

$$
\begin{aligned}
& \rho(\phi)=\rho_{\mathrm{g}}+\left(\rho_{\mathrm{p}}-\rho_{\mathrm{g}}\right) H(\phi), \\
& \eta(\phi)=\eta_{\mathrm{g}}+\left(\eta_{\mathrm{p}}-\eta_{\mathrm{g}}\right) H(\phi)
\end{aligned}
$$

where $H$ denotes the Heaviside function:

$H(\phi)= \begin{cases}1 & \text { if } \phi>0 \\ 0 & \text { if } \phi<0\end{cases}$
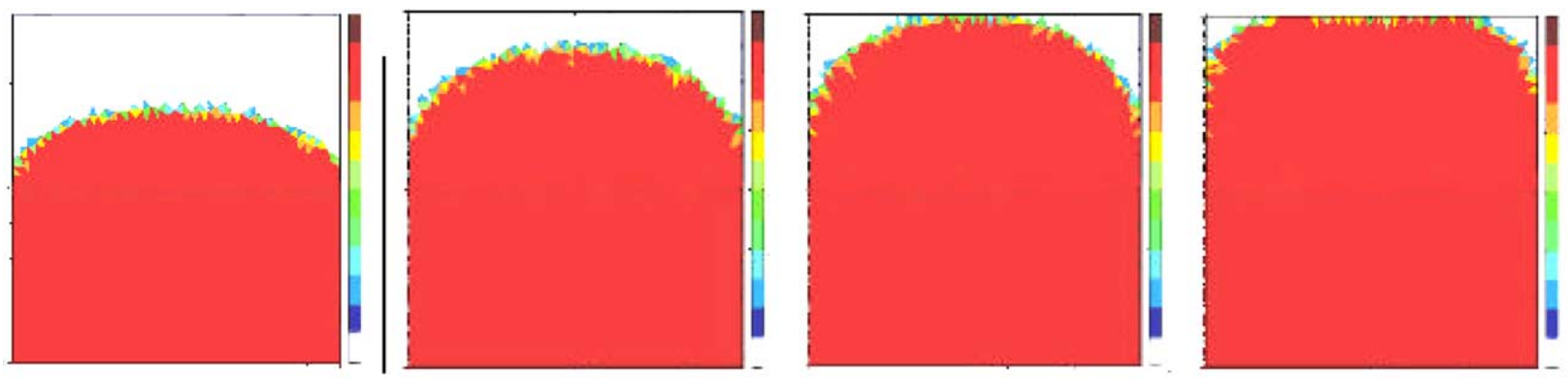

Fig. 2. Foam presence function evolution during a homogeneous expansion in a closed cavity. 
Thus, the motion equation results

$$
\begin{aligned}
& -\int_{\Omega} p \operatorname{Div} \underline{v}^{*} \mathrm{~d} \Omega+2 \int_{\Omega} \eta(\phi) \underline{\underline{D}}: \underline{\underline{D}}^{*} \mathrm{~d} \Omega \\
& \quad=\sigma \int_{\Omega} H(\phi) \operatorname{Grad} k \underline{v}^{*} \mathrm{~d} \Omega+\sigma \int_{\Omega} H(\phi) k \operatorname{Div} \underline{v}^{*} \mathrm{~d} \Omega
\end{aligned}
$$

where $\sigma$ is the surface tension and $k$ the curvature. Taking a smooth approximation of the Heaviside function, Eq. (23) can be written as

$$
\begin{aligned}
& -\int_{\Omega} p \operatorname{Div} \underline{v}^{*} \mathrm{~d} \Omega+2 \int_{\Omega} \eta(\phi) \underline{\underline{D}}: \underline{\underline{D}}^{*} \mathrm{~d} \Omega \\
& =-\sigma \int_{\Omega} k \underline{v}^{*} \operatorname{Grad} H(\phi) \mathrm{d} \Omega
\end{aligned}
$$

where the integral in the second member is not zero only in the interface neighbourhood.

If an explicit discretisation is considered, Eqs. (18) and (19) can be solved using some numerical technique appropriate to its non-linear and hyperbolic character, whereas for solving Eq. (24) a mixed finite element method verifying the LBB condition can be accurately applied. To solve the advection problems (Eqs. (18) and (19)) we use the Euler or the Adams-Bashforth methods for time discretisation and a second order ENO for space discretisation. In [19] several numerical schemes proposed in the literature have been analysed for the resolution of Eq. (19). In this first analysis, the movement of some bubbles into a Newtonian fluid has been simulated. In order to illustrate the capabilities of this technique we depict in Fig. 3 a sequence of a gas bubble deformation under gravity effects into a square closed cavity fulfilled with a Newtonian fluid, where a viscosity relation of 1000 and a density relation of 100 between both fluids are considered. In this example the inertia term in the momentum equation is introduced as well as the gravity effects. The inertia term is stabilised in the numerical discretisation by using the expression of the material derivative along the characteristic curve.

\section{Updated Lagrangian simulation}

\subsection{The natural element method}

In the last decade considerable research efforts have been paid to the development of a series of novel numerical tools that have been referred as meshless or meshfree methods. These methods do not need explicit connectivity information, as required in standard FEM. The geometrical information is generated in a process transparent to the user, alleviating the pre-processing stage of the method. They also present outstanding advantages in modelling complex phenomena, such as large deformation problems, forming processes, fluid flow, etc., where traditional and more experienced techniques, like the FEM, fail due to the need of excessive remeshing. The natural element method (NEM) is one of the latest meshless technique applied in the field of linear elastostatics $[11,12]$. It has unique features among meshless Galerkin methods, such as interpolant character of shape functions and exact imposition of essential boundary conditions. These, and its inherent meshless structure, make the NEM an appealing choice also for application in the simulation of fluid flows. The NEM is based on the natural neighbour interpolation scheme [20,21], which turn relies on the concepts of Voronoi diagrams and Delaunay triangulations (see Fig. 4), to build Galerkin trial and test functions. These are defined as the natural neighbour coordinates (also known as Sibson's coordinates) of the point
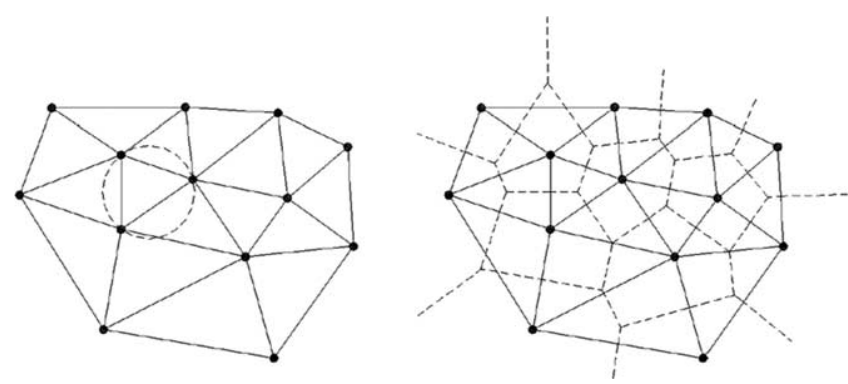

Fig. 4. Delaunay tessellation and Voronoi diagram.

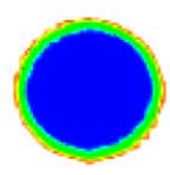

(a)

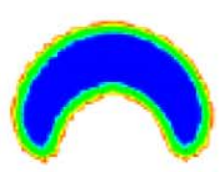

(b)

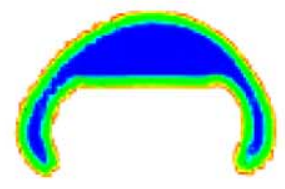

(c)

Fig. 3. Bubble deformation into Newtonian fluid filling a closed cavity and initially at rest: (a) $t=0.1 \mathrm{~s}$, (b) $0.4 \mathrm{~s}$, (c) $0.7 \mathrm{~s}$. 


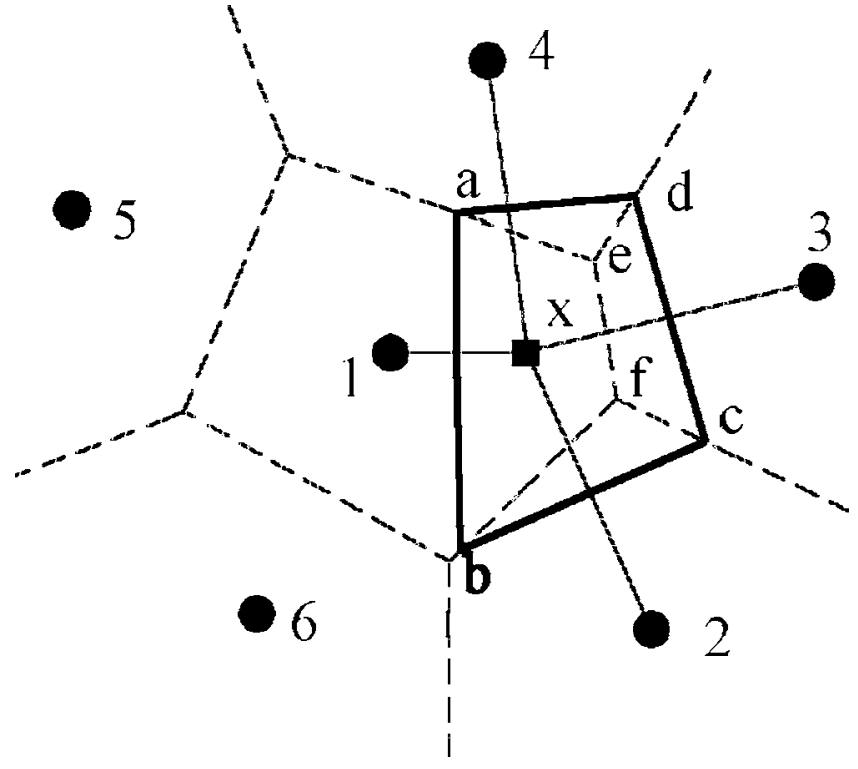

Fig. 5. Natural neighbour interpolants.

under consideration, that is, with respect to Fig. 5, the value at point $x$ of the shape function associated with the node 1 is defined as

$\phi(x)=\frac{\text { Area }(\text { abfe })}{\text { Area }(\text { abcd })}$

These functions are used to build the discrete system of equations arising from the application of the Galerkin method in the usual way. It has been proved [11] that angles of the Delaunay triangulation are not influencing the quality of the results, in opposition to the FEM. In addition, the NEM has interesting properties such as linear consistency and smoothness of the shape functions (natural neighbour coordinates are $C^{1}$ everywhere except at the nodes, where they are $C^{0}$ ). But perhaps the most interesting property of the Natural Element Method is the Kronecker delta property, i.e. $\phi_{i}\left(x_{j}\right)=\delta_{i j}$. In opposition to the vast majority of meshless methods, the NEM shape functions are strictly interpolants. This property allows an exact reproduction of linear (even bilinear in some 3D cases) displacement fields on the boundary of convex domains, since the influence of

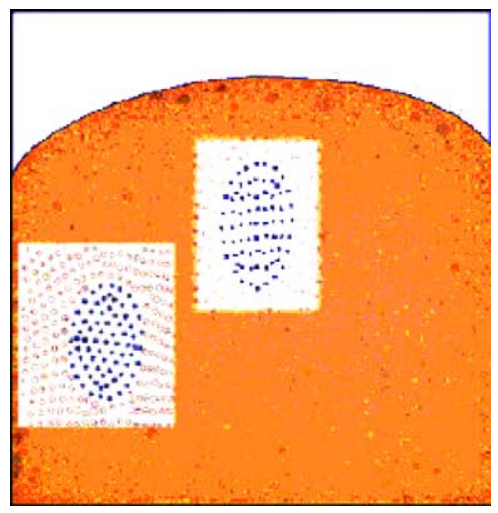

Fig. 7. Cellular shape prediction.

interior points vanishes along convex boundaries. This is not true in non-convex ones, where some specific treatment is required $[12,13]$.

The application of the NEM to complex fluid flow simulations has been recently treated in [22]. The main advantage of using the NEM in the framework of an updated Lagrangian formulation for simulating free or moving surface flows is the fact that the nodal position can be updated from the flow kinematics, without remeshing requirements.

\subsection{Macroscopic level}

In this case the mixed variational formulation (Eqs. (8) and (9)) is discretised by using the natural neighbour interpolation to construct both trial and test functions. In order to describe accurately the mass conservation, a discontinuous pressure approximation considering the pressure constant into each Voronoi cell is used. Fig. 6 depicts the foam expansion sequence corresponding to one shown in Fig. 2.

\subsection{Microscopic level}

In this case, the interface is represented by a set of nodes whose position evolves in time (updated Lagrangian method). The approximations can represent some field discontinuities through the interface using for example the strategies proposed in [13,23]. Thus, in Fig. 7, an example of bubble shape evolution in the microscopic scale is depicted.
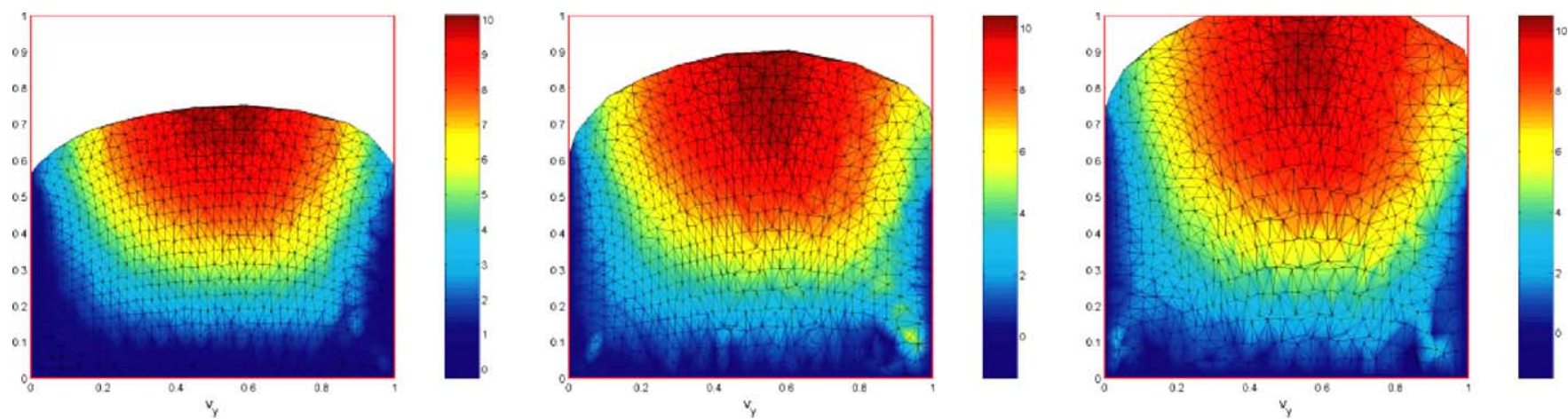

Fig. 6. Sequence of a foam expansion: velocity norm prediction using a meshless simulation. 


\section{Conclusions}

Different simulation strategies able to predict the evolution of the microstructure during foam expansion have been described. The first one concerns a fixed mesh simulation based on the application of the finite element method involving some stabilisations to treat the hyperbolic character of advection terms. The second alternative lies on the use of a meshless technique, in the context of an updated Lagrangian strategy. A deep comparison between both techniques is a work in progress.

\section{References}

[1] P. Chirivella, F. Chinesta, M. Godet, Experimental analysis of the induced anisotropy in foam forming processes, Internal Report, LRTMM-CNAM, Paris, 2000.

[2] M. Sussman, P. Smereka, S. Osher, A level set approach for computing solutions to incompressible two-phase flows, J. Comput. Phys. 114 (1994) 146-159.

[3] M. Sussman, A. Almgren, J. Bell, P. Colella, L. Howell, M. Welcome, An adaptative level set approach for incompressible two-phase flows, J. Comput. Phys. 148 (1999) 81-124.

[4] M. Sussman, E. Fatemi, An efficient interface preserving level set redistancing algorithm and its applications to interfacial incompressible fluid flows, SIAM J. Sci. Comput. 20 (4) (1999) 11651191.

[5] M. Sussman, E. Puckett, A coupled level set and volume-of-fluid method for computing 3D and axisymmetric incompressible two-phase flows, J. Comput. Phys. 162 (2000) 301-337.

[6] N. Sukumar, D. Chopp, N. Moes, T. Belytschko, Modeling holes and inclusions by level sets in the extended finite element method, Comput. Meth. Appl. Mech. Eng. 190 (2001) 6183-6200.

[7] J.M. Melenk, I. Babuska, The partition of unity finite element method: basic theory and applications, Comput. Meth. Appl. Mech. Eng. 39 (1996) 289-314.

[8] F. Chinesta, Predicting material defects in reactive polymeric systems, Int. J. Form. Process. 5 (2-3) (2002) 259-268.
[9] C. Hirt, B. Nichols, Volume of fluid method for the dynamics of free boundaries, J. Comput. Phys. 14 (1974) 227-253.

[10] T. Belytschko, Y. Kronggauz, D. Organ, M. Fleming, Meshless methods: an overview and recent developments, Comput. Meth. Appl. Mech. Eng. 139 (1996) 3-47.

[11] N. Sukumar, B. Moran, T. Belytschko, The natural elements method in solid mechanics, Int. J. Numer. Meth. Eng. 43 (1998) 839-887.

[12] E. Cueto, M. Doblaré, L. Gracia, Imposing essential boundary conditions in the natural elements method by means of density-scaled alpha-shapes, Int. J. Numer. Meth. Eng. 49 (2000) 519-546.

[13] J. Yvonnet, Ph. Lorong, D. Ryckelynck, F. Chinesta, Interpolation naturelle sur les domains non-convexes par l'utilisation du diagramme de Voronoï contraint: méthode des éléments C-naturels, Revue Europeenne des Elements Finis 12/4 (2003) 487-509.

[14] E.B. Richter, C.W. Macosko, Viscosity changes during the isothermal and adiabatic network polymerization, Polym. Eng. Sci. 20 (14) (1980) 921-924.

[15] L. Lefebvre, R. Keunings, Mathematical simulation of the flow of chemically reacting polymeric foams, in: Proceedings of the International Conference on Mathematical Modelling for Material Processing, Clarendon Press, Oxford, 1993, pp. 399-417.

[16] N.S. Deshpande, M. Barigou, Foam phenomena in sudden expansion and contractions, Int. J. Multiphase Flow 27 (2001) 1463-1477.

[17] O. Pironneau, Finite Methods for Fluids, Wiley, 1989.

[18] F. Chinesta, T. Mabrouki, A. Ramon, Some difficulties in the flow front treatment in fixed mesh simulations of composites forming processes, in: Proceedings of the Fifth International Esaform Conference, Krakow, 2002.

[19] P. Quintela, J. Paredes, F. Chinesta, Numerical computation of bubbles motion: a level-set/finite elements methodology, in: Proceedings of the Sixth International Esaform Conference, Salerno, 2003.

[20] R. Sibson, A vector identity for the Dirichlet tesselations, Math Proc. Camb. Phil. Soc. 87 (1980) 151-155.

[21] D.F. Watson, Computing the $n$-dimensional Delaunay tessellation with application to Voronoï polytopes, The Comput. J. $24-2$ (1981) 167-172.

[22] M.A. Martinez, E. Cueto, M. Doblare, F. Chinesta, A meshless simulation of injection processes involving short fibers molten composites, Int. J. Form. Process. 4 (3-4) (2001) 217-236.

[23] E. Cueto, N. Sukumar, B. Calvo, J. Cegoñino, M. Doblaré, Overview and recent developments in Natural Neighbour Galerkin methods, Arch. Comput. Meth. Eng. 10/4 (2003) 307-384. 